\title{
Asymptotic Expansion for Coupled Consolidation
}

\author{
John C.-C. Lu ${ }^{1, *}$ and Feng-Tsai Lin $^{2}$ \\ ${ }^{1}$ Department of Civil Engineering, Chung Hua University, No. 707, Sec. 2, Wufu Rd., Hsinchu 30012, Taiwan R.O.C. \\ ${ }^{2}$ Department of Naval Architecture and Ocean Engineering, National Kaohsiung University of Science and Technology, No. 142, \\ Haijhuan Rd., Kaohsiung 81157, Taiwan R.O.C. \\ ${ }^{*}$ Corresponding author
}

\begin{abstract}
The asymptotic expansion method is used to derive the solutions of a homogeneous isotropic strata subjected to uniform loading on the ground surface. The study develops a mathematical model for the excess pore fluid pressure and land deformation of the strata. Analytical solutions are derived through the application of Laplace transform with respect to the variable of time. The results can improve understanding of the asymptotic expansion theory on coupled consolidation.
\end{abstract}

Keywords-asymptotic expansion; coupled consolidation; Laplace transform; analytical solution

\section{INTRODUCTION}

Responses of strata due to ground surface loading is an important engineering issue. Regarding impact on engineering safety, many studies were concentrated on mechanical and hydraulic behavior due to coupled consolidation. Hydraulic disturbance usually results in a volumetric change of fluid and solid skeleton. The volumetric change can increase excess pore fluid pressure and lead to decrease in effective stress. The loss of shear resistance of solid skeleton may result in a hydraulic failure in the strata. The simulation and its validation are major concern for the safety improvement of the engineering construction.

Klevtsovskiy and Mel'nyk [1] presented asymptotic expansion for the solution of a boundary-value problem in thin cascade domain with a local joint. In the study of Nasution et al. [2], thermomechanical properties and stress analysis of 3-D textile were evaluated by asymptotic expansion homogenization method. Abdel-Halim Hassan [3] addressed the asymptotic expansion and numerical verification method for linear and nonlinear initial value problems. Asymptotic expansion of the Tricomi-Carlitz polynomials and their zeros was presented by Lee and Wong [4]. Parron Vera et al. [5] derived analytical solution of coupled soil erosion and consolidation equations through asymptotic expansion approach. Ho and Fatahi [6] presented one-dimensional consolidation analysis of unsaturated soils subjected to time-dependent loading.

The present investigation is focused on deriving analytical solutions of an isotropic pervious ground space due to uniform loading. The soil or rock mass is modelled as linearly elastic medium with isotropic properties, and the mechanical properties and hydraulic fluid flow are treated as isotropic. Using Laplace integral transform, the analytical solutions of excess pore fluid pressure and displacements of the strata due to uniform loading are obtained.
The results can improve understanding of the surface loading induced coupled responses of the pervious strata.

\section{MATHEMATICAL MODEL}

A. Basic Equations

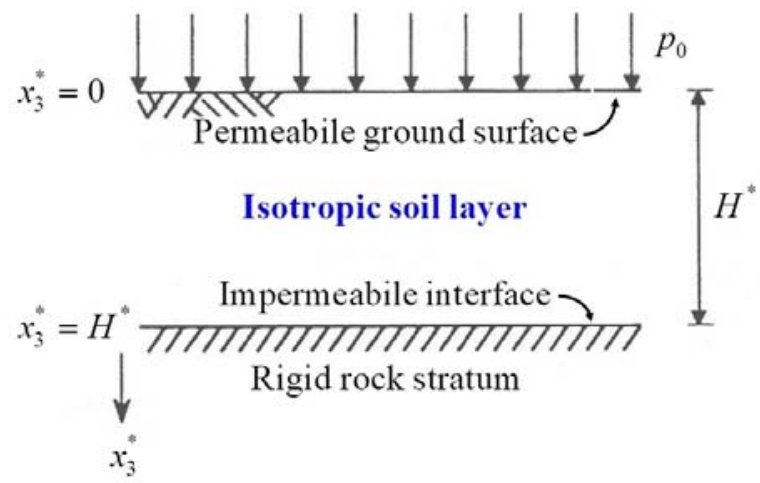

FIGURE I. CONSOLIDATION DUE TO UNIFORM GROUND SURFACE LOADING

Figure 1 displays a surface uniform loading on the pervious porous elastic strata, and the isotropic soil or rock is modeled as a homogeneous elastic medium. The constitutive law for an elastic medium can thus be expressed by

$$
\begin{gathered}
\tau_{i j}^{*}=2 G e_{i j}^{*}+\frac{2 G v}{1-2 v} e^{*} \delta_{i j}-\alpha p^{*} \delta_{i j}, i, j=1,2,3, \\
\theta^{*}=\alpha e^{*}+\frac{p^{*}}{Q},
\end{gathered}
$$

where $\tau_{i j}^{*}, e_{i j}^{*}$, and $p^{*}$ are the total stress components, strain components, and excess pore fluid pressure of the strata, respectively. The increment of fluid volume within unit volume of porous medium is denoted by $\theta^{*}$. The volumetric strain of the porous strata $e^{*}=e_{11}^{*}+e_{22}^{*}+e_{33}^{*}$. The material constants $G$ and $v$ are shear modulus and Poisson's ratio of the isotropic strata. The coupled material constants $\alpha$ and $Q$ are defined by 


$$
\begin{gathered}
\alpha=\frac{3\left(v_{u}-v\right)}{B(1-2 v)\left(1+v_{u}\right)}, \\
\frac{1}{Q}=\frac{9\left(v_{u}-v\right)\left(1-2 v_{u}\right)}{2 G B^{2}(1-2 v)\left(1+v_{u}\right)^{2}},
\end{gathered}
$$

in which $B$ and $v_{u}$ are Skempton pore water pressure coefficient and undrained Poisson's ratio defined by Rice and Cleary [7]. The strain components $e_{i j}^{*}$ are related to displacement components $u_{i}^{*}$ of the strata as below:

$$
e_{i j}^{*}=\frac{1}{2}\left(\frac{\partial u_{i}^{*}}{\partial x_{j}^{*}}+\frac{\partial u_{j}^{*}}{\partial x_{i}^{*}}\right), i, j=1,2,3
$$

In general, the total stresses $\tau_{i j}^{*}$ satisfy the equilibrium equations:

$$
\tau_{i j, j}^{*}+b_{i}^{*}=0, i, j=1,2,3
$$

where $b_{i}^{*}$ denote the body force components. With the effect of body forces neglected, the equilibrium equations can be expressed in terms of displacements and excess pore fluid pressure as follows:

$$
\begin{aligned}
& G \nabla^{2} u_{1}^{*}+\frac{G}{1-2 v} \frac{\partial e^{*}}{\partial x_{1}^{*}}-\alpha \frac{\partial p^{*}}{\partial x_{1}^{*}}=0, \\
& G \nabla^{2} u_{2}^{*}+\frac{G}{1-2 v} \frac{\partial e^{*}}{\partial x_{2}^{*}}-\alpha \frac{\partial p^{*}}{\partial x_{2}^{*}}=0, \\
& G \nabla^{2} u_{3}^{*}+\frac{G}{1-2 v} \frac{\partial e^{*}}{\partial x_{3}^{*}}-\alpha \frac{\partial p^{*}}{\partial x_{3}^{*}}=0,
\end{aligned}
$$

in which the Laplacian $\nabla^{2}=\frac{\partial^{2}}{\partial x_{1}^{* 2}}+\frac{\partial^{2}}{\partial x_{2}^{* 2}}+\frac{\partial^{2}}{\partial x_{3}^{* 2}}$. The fluid flow should obey Darcy's law (6a) and continuity equation (6b) as below:

$$
\begin{aligned}
& v_{i}^{*}=-\frac{k}{\gamma_{w}} \frac{\partial p^{*}}{\partial x_{i}^{*}}, i=1,2,3, \\
& \frac{\partial \theta^{*}}{\partial t^{*}}=-\frac{\partial v_{1}^{*}}{\partial x_{1}^{*}}-\frac{\partial v_{2}^{*}}{\partial x_{2}^{*}}-\frac{\partial v_{3}^{*}}{\partial x_{3}^{*}},
\end{aligned}
$$

in which $v_{i}^{*}$ denotes the fluid volume flow components through a unit area in a set time. The symbols $k$ and $\gamma_{w}$ are permeability and unit weight of pore water of the porous medium. Using Darcy's law (6a) and continuity equation (6b), the governing equation for fluid flow can be derived as:

$$
\frac{k}{\gamma_{w}} \nabla^{2} p^{*}=\alpha \frac{\partial e^{*}}{\partial t^{*}}+\frac{1}{Q} \frac{\partial p^{*}}{\partial t^{*}} .
$$

Equations (5a), (5b), (5c) and (7) govern the responses of the porous medium subjected to ground surface loading. In onedimensional consolidation, the governing equations are simplified as following:

$$
\frac{1}{a} \frac{\partial^{2} u_{3}^{*}}{\partial x_{3}^{* 2}}-\alpha \frac{\partial p^{*}}{\partial x_{3}^{*}}=0,
$$

$$
\frac{k}{\gamma_{w}} \frac{\partial^{2} p^{*}}{\partial x_{3}^{* 2}}=\alpha \frac{\partial^{2} u_{3}^{*}}{\partial x_{3}^{*} \partial t^{*}}+\frac{1}{Q} \frac{\partial p^{*}}{\partial t^{*}} .
$$

The final compressibility $a$ is defined as

$$
a=\frac{1-2 v}{2 G(1-v)} .
$$

\section{B. Boundary Conditions and Initial Conditions}

The uniform load acting on the ground surface is $p_{0}$ as shown in Figure 1, and the study can be treated as onedimensional consolidation model. The soil layer of ground surface boundary $x_{3}^{*}=0$ is regarded as pervious for all times, while the lower boundary at $x_{3}^{*}=H^{*}$ is assumed impervious with a rigid rock shown below:

$$
p^{*}\left(0, t^{*}\right)=0
$$

$$
\frac{\partial u_{3}^{*}\left(0, t^{*}\right)}{\partial x_{3}^{*}}=-a p_{0},
$$

$$
\frac{\partial p^{*}\left(H^{*}, t^{*}\right)}{\partial x_{3}^{*}}=0
$$

$$
u_{3}^{*}\left(H^{*}, t^{*}\right)=0 \text {. }
$$


The initial conditions can be treated as [8]:

$$
\begin{aligned}
& p^{*}\left(x_{3}^{*}, 0^{+}\right)=\frac{a-a_{i}}{\alpha a} p_{0}, \\
& \frac{\partial u_{3}^{*}\left(x_{3}^{*}, 0^{+}\right)}{\partial x_{3}^{*}}=-a_{i} p_{0},
\end{aligned}
$$

where the instantaneous compressibility $a_{i}=a /\left(1+\alpha^{2} a Q\right)$. If the compressibility of solid skeleton and pore water can be neglected, then $\alpha=1, Q \rightarrow \infty$ and $a_{i}=0$ [8].

\section{ASYMPTOTIC EXPANSION}

The non-dimensional characteristic displacement $u_{0}=a \sigma_{0} H^{*}$ and characteristic time $t_{0}=\gamma_{w} H^{* 2} / k \sigma_{0}$, where layered stratum thickness is $H^{*}$, and critical excess pore water pressure $\sigma_{0}=10^{-3} G$ [9]. The space $x_{3}^{*}$, time $t^{*}$, vertical displacement $u_{3}^{*}$ and excess pore water pressure $p^{*}$ can be expressed as

$$
z=\frac{x_{3}^{*}}{H^{*}}, t=\frac{t^{*}}{t_{0}}, w=\frac{u_{3}^{*}}{u_{0}}, p=\frac{p^{*}}{\sigma_{0}},
$$

in which the symbols $z$ and $t$ are dimensionless vertical space variable and dimensionless time variable, respectively; $w$ and $p$ are dimensionless vertical displacement and dimensionless excess pore water pressure, respectively. Therefore, the governing equations of (8a) and (8b) can be transformed to:

$$
\begin{gathered}
\frac{\partial^{2} w}{\partial z^{2}}-\alpha \frac{\partial p}{\partial z}=0 \\
\frac{\partial^{2} p}{\partial z^{2}}-\alpha a \sigma_{0} \frac{\partial^{2} w}{\partial z \partial t}-\frac{\sigma_{0}}{Q} \frac{\partial p}{\partial t}=0 .
\end{gathered}
$$

Besides, the boundary conditions and initial conditions can be expressed as:

$$
\begin{gathered}
p(0, t)=0, \\
\frac{\partial w(0, t)}{\partial z}=-\frac{p_{0}}{\sigma_{0}}, \\
\frac{\partial p(1, t)}{\partial z}=0,
\end{gathered}
$$

$$
w(1, t)=0,
$$

$$
p\left(z, 0^{+}\right)=\frac{a-a_{i}}{\alpha a} \frac{p_{0}}{\sigma_{0}}
$$

$$
\frac{\partial w\left(z, 0^{+}\right)}{\partial z}=-\frac{a_{i} p_{0}}{a \sigma_{0}} .
$$

Using proper asymptotic expansion parameter $\varepsilon=\sigma_{0} / G=10^{-3}$ and asymptotic expansion theory, the onedimensional consolidation settlement and excess pore water pressure can be expressed as

$$
\begin{aligned}
& w=\varepsilon^{0} w^{(0)}+\varepsilon^{1} w^{(1)}+\varepsilon^{2} w^{(2)}+\varepsilon^{3} w^{(3)}+\cdots, \\
& p=\varepsilon^{0} p^{(0)}+\varepsilon^{1} p^{(1)}+\varepsilon^{2} p^{(2)}+\varepsilon^{3} p^{(3)}+\cdots,
\end{aligned}
$$

where $w$ and $p$ are the dimensionless vertical displacement and excess pore water pressure, respectively. The symbols $w^{(i)}$ and $p^{(i)}$ are the ith-order dimensionless vertical displacement and excess pore water pressure with respect to the asymptotic expansion parameter $\varepsilon^{i}$.

\section{A. Basic Equations of Order $\varepsilon^{0}$}

- Governing equations

$$
\begin{gathered}
\frac{\partial^{2} w^{(0)}}{\partial z^{2}}-\alpha \frac{\partial p^{(0)}}{\partial z}=0, \\
\frac{\partial^{2} p^{(0)}}{\partial z^{2}}-\frac{\sigma_{0}}{Q} \frac{\partial p^{(0)}}{\partial t}=0 .
\end{gathered}
$$

- Boundary conditions and initial conditions

$$
p^{(0)}(0, t)=0,
$$

$$
\begin{gathered}
\frac{\partial w^{(0)}(0, t)}{\partial z}=-\frac{p_{0}}{\sigma_{0}}, \\
\frac{\partial p^{(0)}(1, t)}{\partial z}=0,
\end{gathered}
$$




$$
\begin{gathered}
w^{(0)}(1, t)=0, \\
p^{(0)}\left(z, 0^{+}\right)=\frac{a-a_{i}}{\alpha a} \frac{p_{0}}{\sigma_{0}}, \\
\frac{\partial w^{(0)}\left(z, 0^{+}\right)}{\partial z}=-\frac{a_{i} p_{0}}{a \sigma_{0}} .
\end{gathered}
$$

B. Basic Equations of Order $\varepsilon^{1}$

- Governing equations

$$
\begin{gathered}
\frac{\partial^{2} w^{(1)}}{\partial z^{2}}-\alpha \frac{\partial p^{(1)}}{\partial z}=0, \\
\frac{\partial^{2} p^{(1)}}{\partial z^{2}}-\frac{\sigma_{0}}{Q} \frac{\partial p^{(1)}}{\partial t}=\frac{\alpha}{2 \eta} \frac{\partial^{2} w^{(0)}}{\partial z \partial t} .
\end{gathered}
$$

- Boundary conditions and initial conditions

$$
\begin{gathered}
p^{(1)}(0, t)=0, \\
\frac{\partial w^{(1)}(0, t)}{\partial z}=0, \\
\frac{\partial p^{(1)}(1, t)}{\partial z}=0 \\
w^{(1)}(1, t)=0 \\
p^{(1)}\left(z, 0^{+}\right)=0 \\
\frac{\partial w^{(1)}\left(z, 0^{+}\right)}{\partial z}=0 .
\end{gathered}
$$

\section{Basic Equations of Order $\varepsilon^{n}$}

- Governing equations

$$
\begin{gathered}
\frac{\partial^{2} w^{(n)}}{\partial z^{2}}-\alpha \frac{\partial p^{(n)}}{\partial z}=0, \\
\frac{\partial^{2} p^{(n)}}{\partial z^{2}}-\frac{\sigma_{0}}{Q} \frac{\partial p^{(n)}}{\partial t}=\frac{\alpha}{2 \eta} \frac{\partial^{2} w^{(n-1)}}{\partial z \partial t} .
\end{gathered}
$$

- Boundary conditions and initial conditions

$$
p^{(n)}(0, t)=0
$$

$$
\frac{\partial w^{(n)}(0, t)}{\partial z}=0
$$

$$
\frac{\partial p^{(n)}(1, t)}{\partial z}=0
$$

$$
w^{(n)}(1, t)=0
$$

$$
p^{(n)}\left(z, 0^{+}\right)=0,
$$

The model is decoupled as shown in the basic equations of order $\varepsilon^{i}(i=0,1, \cdots, n)$.

\section{ANALYTICAL SOLUTIONS}

Using Laplace transform [10-12], the analytical solutions of mathematical model of order $\varepsilon^{i}$ are derived as $w \cong w^{(0)}+\varepsilon w^{(1)}$ and $p \cong p^{(0)}+\varepsilon p^{(1)}$. Through dedicated calculation, the analytical solutions are derived as:

$$
\begin{aligned}
w(z, t) & \cong \frac{p_{0}}{\sigma_{0}}(1-z)+\frac{p_{0}}{\sigma_{0}} \sum_{n=1}^{\infty} \frac{-4\left(a-a_{i}\right)}{a \pi^{2}}\left[\frac{2}{(2 n-1)^{2}}\right. \\
+\varepsilon & \left.\frac{\pi^{2} \alpha^{2}}{4 \eta} c^{2} t \cos \frac{(2 n-1) \pi z}{2}\right] e^{-\frac{(2 n-1)^{2} \pi^{2}}{4} c t},
\end{aligned}
$$

$$
p(z, t) \cong \frac{2\left(a-a_{i}\right)}{\pi a} \frac{p_{0}}{\sigma_{0}} \sum_{n=1}^{\infty}\left[\frac{2}{\alpha(2 n-1)}+\varepsilon \frac{(2 n-1) \pi^{2} \alpha}{4 \eta} c^{2} t\right]
$$




$$
\times \sin \frac{(2 n-1) \pi z}{2} e^{-\frac{(2 n-1)^{2} \pi^{2}}{4} c t},
$$

where the dimensionless consolidation parameter $c$ and $\eta$ are defined as $c=Q / \sigma_{0}$ and $\eta=(1-v) /(1-2 v)$, respectively. The total settlement can be derived by letting $z=0$ in equation (21a):

$$
\begin{aligned}
w(0, t) \cong \frac{p_{0}}{\sigma_{0}}\left\{\sum_{n=1}^{\infty}\right. & \frac{-4\left(a-a_{i}\right)}{a \pi^{2}}\left[\frac{2}{(2 n-1)^{2}}+\varepsilon \frac{\pi^{2} \alpha^{2}}{4 \eta} c^{2} t\right] \\
& \left.\times e^{-\frac{(2 n-1)^{2} \pi^{2}}{4} c t}+1\right\} .
\end{aligned}
$$

\section{CONCLUSIONS}

Based on asymptotic expansion theory of elasticity of porous media, the coupled mathematical model is decoupled by using proper asymptotic expansion parameter $\varepsilon$ as shown in the mathematical equations. The transient analytical solutions of a homogeneous isotropic elastic layer for one-dimensional deformation and excess pore fluid pressure subjected to uniform loading are presented by equations (21a) and (21b). The results can improve understanding of the asymptotic expansion theory on coupled consolidation.

\section{ACKNOWLEDGMENT}

This work is supported by the Ministry of Science and Technology of Taiwan, Republic of China, through grants NSC89-2211-E-216-032 and MOST106-2625-M-415-003.

\section{REFERENCES}

[1] A. V. Klevtsovskiy, and T. A. Mel'nyk, “Asymptotic expansion for the solution to a boundary-value problem in a thin cascade domain with a local joint,” Asymptotic Analysis, vol. 97, no. 3-4, pp. 265-290, 2016.

[2] M. R. E. Nasution, N. Watanabe, A. Kondo, and A. Yudhanto, "Thermomechanical properties and stress analysis of 3-D textile composites by asymptotic expansion homogenization method," Composites Part B: Engineering, vol. 60, pp. 378-391, 2014.

[3] I. H. Abdel-Halim Hassan, "The asymptotic expansion and numerical verification method for linear and nonlinear initial value problem," Applied Mathematics and Computation, vol. 180, no. 1, pp. 29-37, 2006.

[4] K. F. Lee, and R. Wong, "Asymptotic expansion of the Tricomi-Carlitz polynomials and their zeros," Journal of Computational and Applied Mathematics, vol. 265, pp. 220-242, 2014.

[5] M. A. Parron Vera, F. Yakhlef, M. D. Rubio Cintas, O. Castillo Lopez, P. Dubujet, A. Khamlichi, and M. Bezzazi, "Analytical solution of coupled soil erosion and consolidation equations by asymptotic expansion approach,” Applied Mathematical Modelling, vol. 38, no. 15-16, pp. 4086-4098, 2014.

[6] L. Ho, and B. Fatahi, "One-dimensional consolidation analysis of unsaturated soils subjected to time-dependent loading," International Journal of Geomechanics, vol. 16, no. 2, pp. 1-19, 2016.

[7] J. R. Rice, and M. P. Cleary, "Some basic stress diffusion solutions for fluid-saturated elastic porous media with compressible constitutents," Reviews of Geophysics and Space Physics, vol. 14, no. 2, pp. 227-241, 1976.
[8] M. A. Biot, “General theory of three-dimensional consolidation,” Journal of Applied Physics, vol. 12, no. 2, pp. 155-164, 1941.

[9] M. Kurashige, "A thermoelastic theory of fluid-filled porous materials," International Journal of Solids and Structures, vol. 25, no. 9, pp. 10391052, 1989.

[10] I. N. Sneddon, Fourier Transforms, McGraw-Hill, New York, 1951

[11] I. S. Gradshteyn, and I. M. Ryzhik, Table of Integrals, Series, and Products, Academic Press, New York, 1980.

[12] A. Erdelyi, W. Magnus, F. Oberhettinger, and F. G. Tricomi, Tables of Integral Transforms, McGraw-Hill, New York, 1954.

\section{NOTATION OF SYMBOLS}

$a$

$a_{i}$

C

$e^{*}$

$e_{i j}^{*}$

G

$H^{*}$

$k$

$p$

$p_{0}$

$p^{(i)}$
Final compressibility defined in equation (9), $a=1 / 2 \eta G\left(\mathrm{~Pa}^{-1}\right)$

Instantaneous compressibility, $a_{i}=a /\left(1+\alpha^{2} a Q\right)$ (Dimensionless)

\section{Body force components $\left(\mathrm{N} / \mathrm{m}^{3}\right)$}

Skempton pore fluid pressure coefficient of the isotropic strata (Dimensionless)

Consolidation parameter, $c=Q / \sigma_{0}$ (Dimensionless)

Volumetric strain of the isotropic strata, $e^{*}=e_{11}^{*}+e_{22}^{*}+e_{33}^{*}$ (Dimensionless)

Strain components of the isotropic strata (Dimensionless)

$$
\text { Shear modulus of the isotropic strata }(\mathrm{Pa})
$$

* Thickness of layered stratum (m)

Excess pore water pressure defined in equation (11) (Dimensionless)

Uniform load acting on the ground surface $(\mathrm{Pa})$

The ith-order dimensionless excess pore water pressure with respect to the asymptotic expansion parameter $\varepsilon^{i}$ (Dimensionless)

* Excess pore water pressure of the isotropic strata $(\mathrm{Pa})$

Coupled material constant defined in equation (2b) (Pa)

Dimensionless time variable defined in equation (11) (Dimensionless)

Characteristic time, $t_{0}=\gamma_{w} H^{* 2} / k \sigma_{0}$ (s)

Time variable (s)

Characteristic displacement, $u_{0}=a \sigma_{0} H^{*}(\mathrm{~m})$

Displacement components of the isotropic strata (m)

Fluid volume flow components through a unit area in a set time $(\mathrm{m} / \mathrm{s})$

Vertical displacement defined in equation (11) (Dimensionless) 
$w^{(i)} \quad$ The ith-order dimensionless vertical displacement with respect to the asymptotic expansion parameter $\varepsilon^{i}$ (Dimensionless)

$x_{i}^{*} \quad$ Space variable in $i$-direction (m)

z Dimensionless vertical space variable defined in equation (11) (m)

$\alpha \quad$ Coupled material constant defined in equation (2a) (Dimensionless)

$\gamma_{w} \quad$ Unit weight of pore water $\left(\mathrm{N} / \mathrm{m}^{3}\right)$

$\delta_{i j} \quad$ Kronecker delta function (Dimensionless)

$\varepsilon \quad$ Asymptotic expansion parameter, $\varepsilon=\sigma_{0} / G=10^{-3}$ (Dimensionless)

$\eta \quad$ Parameter, $\eta=(1-v) /(1-2 v)$ (Dimensionless)

$\theta^{*} \quad$ Increment of fluid volume within unit volume of the isotropic strata (Dimensionless)

$v \quad$ Poisson's ratio of the isotropic strata (Dimensionless)

$v_{u} \quad$ Undrained Poisson's ratio of the isotropic strata (Dimensionless)

$\sigma_{0} \quad$ Critical excess pore water pressure, $\sigma_{0}=10^{-3} G(\mathrm{~Pa})$

$\tau_{i j}^{*} \quad$ Total stress components of the isotropic strata (Pa)

$\nabla^{2} \quad$ Laplacian, $\nabla^{2}=\frac{\partial^{2}}{\partial x_{1}^{* 2}}+\frac{\partial^{2}}{\partial x_{2}^{* 2}}+\frac{\partial^{2}}{\partial x_{3}^{* 2}}\left(\mathrm{~m}^{-2}\right)$ 